\section{Representación de la acción política juvenil en redes sociales: Análisis crítico de las prácticas discursivas producidas durante las movilizaciones estudiantiles en Chile (2011-2013)}

Representation of youth political action on social networks: Critical analysis of discursive practices produced during student mobilizations in Chile (2011-2013)

\section{Camila Cárdenas-Neira ${ }^{* *}$}

El artículo presentado se deriva de la investigación doctoral financiada por el Programa de Formación de Capital Humano Avanzado de CONICYT mediante la Beca de Doctorado en el Extranjero Becas Chile Folio № 72130323.

** Grupo de Estudios del Discurso (GED), Departamento de Traducción y Ciencias del Lenguaje, Universidad Pompeu Fabra. Roc de Boronat 138, Barcelona, España. Correo electrónico: camila.cardenas.neira@gmail.com.

\section{Resumen}

Este artículo muestra resultados preliminares de una investigación en curso sobre las prácticas discursivas online producidas por los/as jóvenes chilenos en el contexto de las recientes movilizaciones estudiantiles (2011-2013): la gestión contra-informativa y la expansión transmedia en redes sociales. Por un lado, se constata que los/as jóvenes resisten estratégicamente el tratamiento noticioso de los medios de comunicación tradicionales, disputando la conformación de representaciones sociales críticas sobre su acción colectiva a partir de una reorientación ideológica de las informaciones disponibles sobre su protesta. Por otro lado, se observa que los/as jóvenes producen y difunden un conjunto de discursos multimodales distintivos de sus actuales repertorios de interacción comunicativa, amplificando efectos sociocognitivos relevantes para la conformación de marcos de interpretación colectiva. Ambas prácticas discursivas promueven ejercicios de resignificación sin precedentes en la Web Social, con los cuales los/as jóvenes reivindican sistemáticamente sus identidades, conocimientos y memorias intrageneracionales.

Palabras clave: movimiento estudiantil chileno, prácticas discursivas online, gestión contrainformativa, expansión transmedia, redes sociales.

\begin{abstract}
This paper shows preliminary results of an ongoing investigation about the online discursive practices produced by young Chileans in the
\end{abstract}


context of the recent student mobilizations (2011-2013): counter-information management and transmedia expansion in social networks. On one hand, it is noted that young people resist strategically news coverage of traditional media, disputing the formation of critical social representations of his collective action from an ideological reorientation of available information about their protest. On the other hand, it is observed that young people produce and disseminate a set of distinctive multimodal discourses of their current repertories of communicative interaction, amplifying relevant social and cognitive effects for the formation of collective interpretation frames. Both discursive practices promote unprecedented resignification exercises on the Social Web, with which young people systematically claim their identities, knowledge and intra-generational memories.

Key words: Chilean students' movement, online discursive practices, counter-information management, transmedia expansion, social networks.

\section{Introducción}

Es un hecho que la acción política juvenil se encuentra unida a la historia de los movimientos sociales en Chile (Garcés 2012), en particular su variante estudiantil (Aguilera 2012), patente sobre todo a partir de los procesos de reforma y contra-reforma universitaria entre fines de 1960 y principios de 1980. No obstante, la transición de la dictadura a la democracia en 1990 deja paulatinamente en evidencia los distintos mecanismos materiales (de violencia de Estado) y simbólicos (de representación ideológica) que aseguran la negación, la marginación y el ocultamiento de la juventud en el pasado reciente del país (Cárdenas 2014a).

Con el cambio de siglo, los/as estudiantes chilenos/as comienzan a tensionar estas formas de invisibilización, logrando una figuración inédita particularmente en la última década (2006-2016). Demandas por una educación pública, gratuita y de calidad signan sus recientes ciclos movilizatorios, instalando consigo una crítica mayor al modelo de sociedad neoliberal que, en su conjunto, estructura y profundiza una lógica reproductora de desigualdades heredada por el régimen militar (1973-1990).

En este escenario, el interés por la movilización juvenil de posdictadura se vincula explícitamente con los cambios que han experimentado las nuevas generaciones en relación con sus modos de comprender, identificarse y ejercer la política, a partir del abandono sistemático de las prácticas tradicionales de participación institucionalizada (Cárdenas 2011; Muñoz 2011), y de la creciente adopción de prácticas de culturalización que configuran sus modalidades emergentes de actuación y contestación (Aguilera 2014; Reguillo 2012).

En virtud del aprendizaje acumulado en las últimas movilizaciones, los/as jóvenes son capaces de avanzar "hacia la construcción de discursividades más complejas que van produciendo sentidos políticos sobre sus propias prácticas" (Aguilera 2010a: 85). Este aprendizaje ha impactado en las tácticas de ocupación de los espacios públicos y mediáticos, mostrando, hasta aquí, una doble finalidad: confrontar los modos de representación dominante que los/as sindica como sujetos de violencia, desviación y amenaza, y oponerse a la autoridad gubernamental y policial que los/as coacciona 
y constriñe. Todo esto ha repercutido en las formas de ritualización del conflicto educativo (Aguilera 2014), dando lugar a un tipo de movilización colectiva lúdica (Aguilera 2010b) que no sólo optimiza los procesos de afirmación identitaria, afiliación grupal y cohesión interna, sino que coordina subjetividades específicas de un movimiento que propone a la ciudadanía otra racionalidad y otra imaginación (Pino-Ojeda 2014).

Nada de esto sería posible si la comunicación no constituyera un elemento clave en el surgimiento de nuevas prácticas políticas (Martín Rojo 2013), de modo que la interconexión de los espacios offline y online supone una transformación paradigmática dada por el desplazamiento de los tradicionales repertorios de acción colectiva a los actuales repertorios de interacción en red. De esta manera, los/as estudiantes se instituyen como un movimiento social reflexivo (Martín Rojo 2016), que gestiona sus prácticas discursivas como un bien estratégico para la resistencia contra-hegemónica tanto dentro como fuera de la web.

$\mathrm{Si}$ en el actual contexto tecnológico el movimiento estudiantil apropia múltiples medios y modos expresivos con el fin de posicionar su protesta públicamente, conviene preguntarse qué aspectos inciden en la representación de su participación política, especialmente en plataformas de interacción online donde los/as propios/as jóvenes se perfilan como principales mediadores/as comunicativos/as. En esta dirección, estudiar las prácticas discursivas juveniles en redes sociales busca no sólo profundizar en la base socio-semiótica que fundamenta su producción de sentidos en torno a la crisis educativa, sino que, además, llama a poner atención en la base socio-cognitiva que modela identidades, conocimientos y memorias imprescindibles para dar sustento ideológico a sus acciones e intercambios colectivos.

Analizar los usos del lenguaje en las redes sociales permite dar cuenta de las reubicaciones que experimentan los espacios de enunciación en los que se emplazan los actores juveniles, controlando discrecionalmente la elaboración y la circulación de toda clase de productos comunicativos con los que tornan inteligible su movilización (Cárdenas 2014b). Éstos se desprenden de -y pueden dar forma a- varias prácticas contenciosas que emplean internet y aplicaciones móviles para su planificación y propagación, tales como la protesta callejera con marchas, funas y performances registradas mediante fotografías y vídeos, la contestación lingüística en base a lienzos, pancartas y rayados, y la creación gráfica de diseños como memes, caricaturas, afiches, etc. Dichos productos a menudo se desplazan a través de distintos espacios públicos y mediáticos de manera simultánea, pudiendo articular medios y modos semióticos diferentes, transponer significados convergentes $\mathrm{O}$ divergentes entre sí, y formular representaciones sociales competitivas o alternativas.

En esta línea, esta investigación se ha propuesto analizar cómo los/as jóvenes emplean las redes sociales para construir, poner en circulación e interpretar discursos que son constitutivos de su acción política, a partir de los cuales pueden discrepar y posicionarse como interlocutores legítimos en el debate sobre la educación en Chile. Esta opción distingue este trabajo de otros que han abordado la representación del movimiento estudiantil exclusivamente desde los discursos hegemónicos, o bien, han atendido sus estrategias tecno-políticas con 
escaso interés por los significados construidos en las interacciones comunicativas llevadas a cabo en la Web Social.

Para dar cuenta de esta aproximación, a continuación se plantea un marco teórico de enfoque interdisciplinar, sustentado principalmente en las aportaciones de los Estudios del Discurso, el Análisis del Discurso Mediado por Computadora y la Semiótica Social. Luego se presenta el diseño de investigación empleado para identificar, caracterizar y explicar las prácticas de gestión contra-informativa y expansión transmedia examinadas hasta ahora. Posteriormente se exponen estas prácticas y se provee una propuesta provisoria de análisis y algunos ejemplos recolectados de dos cuentas de Facebook exploradas. Finalmente, se puntualizan algunas conclusiones que permiten sintetizar sus funciones discursivo-semióticas, sociocognitivas e histórico-políticas más prominentes.

\section{Marco teórico}

En los últimos años se han incrementado las investigaciones que buscan relevar los aspectos políticos, históricos y culturales distintivos de los actuales movimientos sociales en red (Feixa, Fernández-Planells y Figueras-Maz 2016; Castells 2014, 2012; Bennett y Segerberg 2013; Tarrow 2011; Tilly y Wood 2010; Feixa, Pereira y Juris 2009). Sin embargo, son todavía pocos los estudios que se han enfocado en el rol que los medios y las prácticas comunicativas desempeñan en estos movimientos (CostanzaShock 2014, 2013, 2012; Cammaerts, Mattoni y McCurdy 2013; Castells 2009, 2007).

Desde fines del siglo $X X$ se han propuesto diversas etiquetas que intentan abarcar la relación entre tecnología y acción colectiva: clickactivismo (Peña-López 2013; Butler 2011), hacktivismo (Jordan y Taylor 2004; Samuel 2004), ciberactivismo (Tascón y Quintana 2012; McCaughey y Ayers 2003), ciberprotesta (Van de Donk et al. 2004; Pickerill 2003), ciberpolítica (Bell 2006; Hill y Hughes 1998) y tecnopolítica (Toret 2015; Toret et al. 2013) son algunas de las más frecuentes. En estas propuestas, la comunicación recibe una mención constante pero ambigua, generalmente referencial o dada por sentada.

Hasta la fecha, la producción académica ha tendido a disociar los ámbitos de sociabilidad online y offline de los movimientos sociales, de modo que al compararse el impacto de las actividades gestadas dentro y fuera de la web se han polarizado sus beneficios y limitaciones. En este sentido, el énfasis ha estado puesto en cómo los usos tecnológicos traducen los rendimientos políticos, evaluándose su nivel de dependencia y eficacia. De esta manera, se han disgregado: a) los usos más bien prácticos vinculados con la organización de eventos multitudinarios, el establecimiento de tácticas de ocupación y resistencia contra la represión policial y la vigilancia gubernamental, y la obtención de información acerca de las protestas en curso; de b) los usos más bien figurativos asociados con la identificación de los miembros y la diferenciación de los adversarios, la reflexión acerca de los asuntos que resultan relevantes para asegurar la cohesión grupal, y la discusión en torno a los lineamientos ideológicos que enmarcan las estrategias de interpretación y acción colectiva. Cuando estos usos se asumen en conjunto, el resultado suele reducirse a la sumatoria de sus partes, sin que se establezcan vínculos significativos entre ellos como un todo articulado (Cárdenas 2014c). 
En este contexto, si bien se reconoce que los discursos que los actores movilizados emplazan y despliegan en la web cumplen un papel destacado, resultan escasos los análisis que puntualizan sus condiciones de producción, circulación y comprensión. Es innegable, además, que las transformaciones sociales derivadas de los procesos de movilización ciudadana a nivel global han coincidido con diversos cambios en las prácticas comunicativas soportadas tecnológicamente, las que incluyen, combinan y condensan una multiplicidad de sistemas sígnicos, modos semióticos y recursos lingüísticos que operan simultáneamente (Pardo 2012a), con rendimientos cada vez más sofisticados y complejos.

Las investigaciones en el ámbito de las ciencias sociales, en general, y de las ciencias del lenguaje, en particular, se enfrentan al desafío de distinguir e interpretar los actuales discursos mediáticos, caracterizados por hibridar medios y herramientas digitales que coexisten en expresiones cuyos límites aún no están establecidos de forma clara. En particular, la Web Social da un nuevo carácter comunicativo a los procesos de construcción colectiva e intercambio masivo de discursos contra-hegemónicos (Pardo 2012a), los que, a su vez, modifican las formas de producción, distribución y estabilización de significados potencializados por la utilización de Internet (Kress 2010) en entornos colaborativos.

En el ámbito de los Estudios del Discurso, propuestas procedentes del Análisis del Discurso Mediado por Computadora (Herring y Androutsopoulos 2015; Herring 2014, 2013, 2004) proveen la noción de discurso 2.0 para identificar aquellos textos creados y difundidos específicamente en redes sociales, cuyo análisis involucra cuatro niveles interrelacionados: estructural, semánticopragmático, interaccional y social. A grandes rasgos, estos textos comportan distintos grados de familiaridad, reconfiguración y emergencia que no necesariamente afectan su constitución interna, es decir, su organización discursiva propiamente tal, sino que, más bien, propenden al establecimiento de nuevos contenidos, contextos y patrones de uso e interacción.

Desarrollos en el campo de la Semiótica Social (Kress 2010; Martinec y Van Leeuwen 2008; Machin y Van Leeuwen 2007; Kress y Van Leeuwen 2006, 2001; Van Leeuwen 2005) muestran que los significados se construyen de forma motivada y situada histórica, política y culturalmente a partir de diferentes modos comunicativos (i.e. escrito, visual, sonoro, etc.) que, en combinación, configuran los discursos mediáticos contemporáneos. A su vez, cuando estos procesos de construcción de significados se llevan a cabo en la web nunca se derivan de un único medio (Lehtonen 2000), propiciando fenómenos de intermedialidad (Elleström 2010) que dejan de manifiesto la interconexión de discursos multimodales a través de variadas plataformas.

Ambas aproximaciones permiten analizar cómo estos significados confluyen y se acumulan de acuerdo a las rutas de navegación que siguen los/as usuarios/as de Internet, superando las delimitaciones convencionales entre espacios, tiempos y prácticas sociales (Lemke 2009a, 2009b). Al respecto, dos procesos de significación se tornan relevantes: uno es el de hipermodalidad (Lemke 2003), útil para distinguir la naturaleza multisemiótica de estas trayectorias hipertextuales, y el otro es el de resemiotización (ledema 2003), útil para esclarecer cómo las comunidades online 
transponen y reubican sus saberes, identidades y posicionamientos personales y grupales cuando se desplazan sucesivamente de unos entornos mediáticos a otros.

Estos procesos se fundan en un principio de inconmensurabilidad, a partir del cual el potencial de significado de un modo comunicativo es transversalmente contextualizado en base a las características del medio tecnológico en el que aparecen los discursos (Lemke 2005). Por una parte, los/as usuarios/as de Internet construyen determinadas cadenas semánticas a través de distintos medios "no tanto por su referencia a un significado común (...), como a una consonancia de apelaciones a sus disposiciones interpretativas" (Lemke 2009a: 587), es decir, enlazan unos discursos con otros a partir de los puntos en común que les parecen relevantes. Además, esto permite explicar "cómo y por qué los significados que hacemos aquí y ahora están conectados a los realizados por otros con los que formamos una red de interdependencia mutua" (Lemke 2001: 80), de modo que estos procesos interpretativos pueden incluir la participación de distintos/as usuarios/as a la vez.

Por otra parte, Lehtonen (2000) retoma la teoría de la intertextualidad para recalcar que estos discursos son leídos tanto en relación con otros discursos que circulan en la web, como en relación con el conocimiento textual poseído por sus usuarios/as, conformando trayectorias de sentido determinadas. En su propuesta, tales trayectorias pueden analizarse a partir de dos ejes: intertextualidad horizontal e intertextualidad vertical. El primero incluye vínculos entre textos primarios que abarcan temas, participantes y referentes comunes. El segundo prevalece entre un texto primario $y$ otros textos secundarios que se refieren explícita o implícitamente a él. De esta forma, se crean textos terciarios a partir de los cuales los/as lectores/as extraen, enlazan y negocian el contenido social, político, cultural e histórico de estos discursos conjuntamente.

Esta revisión permite trazar relaciones entre perspectivas disciplinares complementarias que reparan en el carácter multimodal, intermedial e intertextual de las prácticas comunicativas online, que en este caso podemos aplicar al análisis de los discursos elaborados y difundidos en redes sociales por los/as jóvenes en el contexto de sus recientes movilizaciones. A medida que el movimiento estudiantil se apropia de plataformas multimedia más complejas, como es el caso de Facebook, Twitter, Youtube y otras afines, mayor preponderancia adquieren los efectos ideológicos y epistémicos que modelan los procesos de producción, circulación e interpretación discursiva en la Web Social. En este punto, resulta indispensable analizar cómo se co-articulan tales procesos en la formulación de representaciones sociales concretas, esto es, dilucidar cómo se construyen y fundamentan los marcos o esquemas de pensamiento que dan significado a sus modos de acción, identificación e interpretación colectiva (Snow 2004; Benford y Snow 2000; Gamson y Meyer 1999; McAdam, McCarthy y Zald 1999; Snow et al. 1989).

En suma, los usos del lenguaje en la Web Social son esenciales para mediar la acción y la comunicación de los actuales movimientos en red (Cammaerts, Mattoni y McCurdy 2013), de allí que resulte de interés dar cuenta de los procesos semióticos que posibilitan las interacciones de sus miembros a diferentes escalas espaciales y temporales. Esta aproximación supondría la existencia de unos principios de significación compartidos al interior del grupo, los cuales, a su 
vez, requerirían de la coordinación de distintas formas de cognición personal y social que darían sustento a estos intercambios simbólicos.

\section{Proyecto de investigación}

\subsection{Diseño de investigación}

Este diseño se constituyó sobre la base de un proceso iterativo de decisiones sobre:
1) las preguntas y los objetivos del estudio, y 2) la conformación de un marco teórico y un plan metodológico que resultaran apropiados para satisfacerlas/os. Siguiendo a Page et al. (2014), se modelaron etapas progresivas que permitieran comprender el problema desde una perspectiva integrada y flexible, esto es, atendiendo los desafíos de analizar las prácticas discursivas producidas en redes sociales de acuerdo a su carácter dinámico y mutable. Dichas etapas se esquematizan en la Figura 1.

Figura 1. Síntesis de las etapas del diseño de investigación

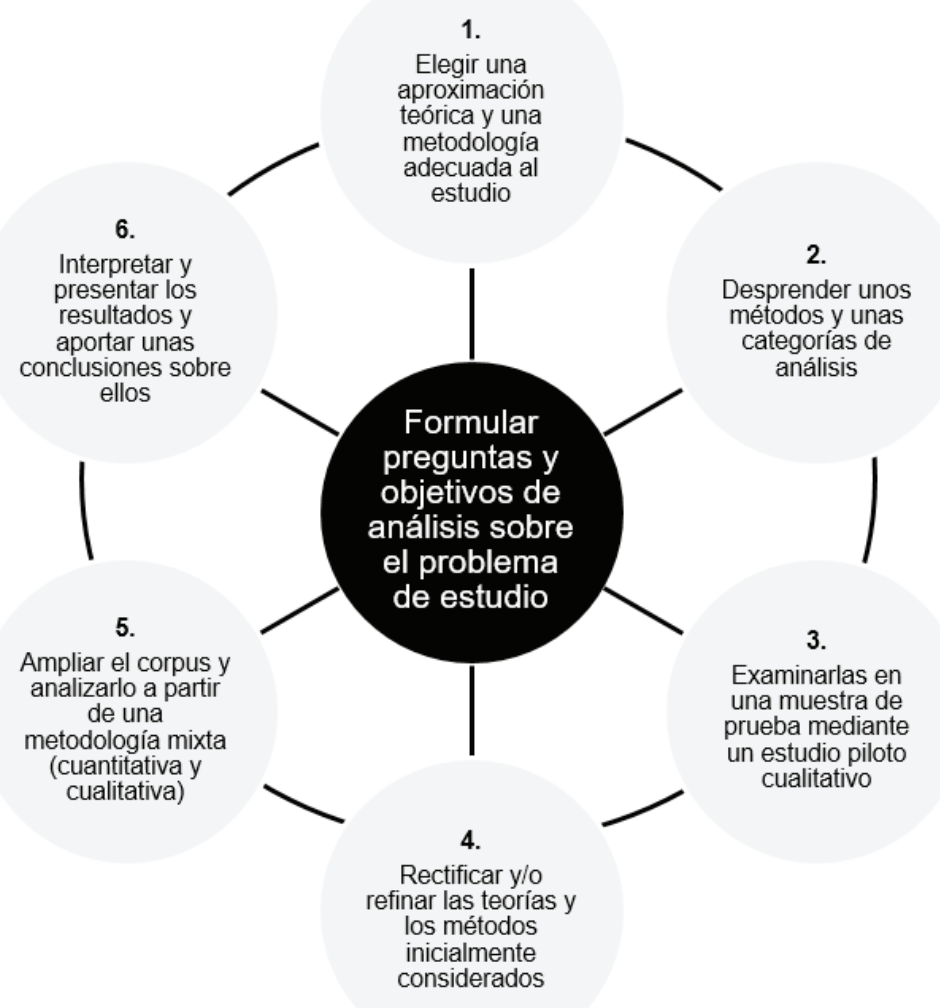

Fuente: elaboración propia a partir de las directrices de Page et al. (2014). 
Durante el primer año de investigación (2013), los análisis de algunas muestras de prueba ayudaron a identificar un tipo de práctica discursiva que resultaba prominente hasta entonces, a saber, la gestión de contenidos periodísticos sobre las movilizaciones al interior de las comunidades estudiantiles online. Básicamente, se observó que la mayor parte de las publicaciones diarias se basaba en la reposición de noticias tomadas de distintos medios de comunicación nacionales (prensa, radio y televisión), en virtud de lo cual se propiciaban ricas instancias de discusión juvenil a partir de los comentarios que efectuaban los/ as seguidores/as sobre dichos contenidos.

No obstante, hacia el segundo año de investigación (2014), estos análisis de prueba hicieron posible distinguir otro tipo de práctica discursivaque adquiría cada vez mayor presencia en redes sociales, basada, específicamente, en la producción y la circulación de diversos discursos de autoríajuvenil ${ }^{1}$. Dicho de otro modo, las publicaciones no sólo se configuraban a partir de discursos ajenos sobre las acciones del movimiento estudiantil, sino que gradualmente se incorporaban discursos construidos desde los actores y colectivos juveniles.

A la luz de estos primeros análisis, en el tercer año de investigación (2015) tanto las preguntas y los objetivos como las aproximaciones teóricas y metodológicas fueron ajustándose y precisándose consecutivamente. Por estas razones, el diseño original sufrió distintas modificaciones con el pasar del tiempo.

Dado que, en muchos casos, es complejo determinar fehacientemente la autoría de los textos que circulan en redes sociales, cuando se alude a discursos propios se considera en un sentido amplio a aquellos cuyos contenidos se alinean con el movimiento estudiantil, esto es, que auto-representan la acción política juvenil desde la perspectiva del endogrupo, contando sus productores/as como miembros o aliados/as.
Actualmente se ha concluido la cuarta etapa, lo que permite adelantar algunos hallazgos preliminares y delinear ciertas reorientaciones a futuro? ${ }^{2}$.

\subsection{Conformación del corpus}

En el proyecto de investigación inicial se seleccionó un conjunto de cuentas de Facebook que se caracterizaban por: 1) publicar mayoritariamente contenidos sobre el movimiento estudiantil (en ocasiones se hacía referencia a otros movimientos ciudadanos afines); 2) dirigirse principalmente a un segmento juvenil; 3) alcanzar un alto número de seguidores/as (similar al de los principales medios nacionales de prensa con presencia en redes sociales), y; 4) mantenerse vigentes durante el periodo de estudio considerado. En la Figura 2 se sintetizan los principales criterios que definieron la conformación del corpus.

De las cuentas consideradas, se priorizaron dos de las más populares. La primera se denomina Estudiantes Informados (El de ahora en adelante), la cual contaba con más de 150.000 seguidores/as al 2013. La segunda se denomina Universitario Informado (UI de ahora

\footnotetext{
Al llegar a la etapa cinco y ampliar el corpus de estudio surge la necesidad de refinar aún más el modelo de análisis sugerido más adelante. Principalmente, cuando se ponen a prueba las categorías seleccionadas se observa que éstas todavía resultan generales si se aplican a un repertorio más amplio de géneros discursivos. En este sentido, sobresalen dos reorientaciones fundamentales. Una investigación de esta naturaleza resulta más funcional si se ciñe a criterios de representatividad más que de exhaustividad, esto es, si se privilegia un análisis más delicado de unidades distintivas en lugar de un análisis más general de todos los ejemplares disponibles. En esta dirección, es crucial contar, primero, con una mejor caracterización del macrogénero "publicación" en Facebook, y desde esa estructura derivar posteriormente los patrones de construcción de significados -a distintos niveles de realización (discursivo, semiótico, ideológico y epistémico)- que son particulares de cada género (noticias, caricaturas, memes, afiches, fotografías, etc.).
} 
Figura 2. Síntesis de los principales criterios de conformación del corpus

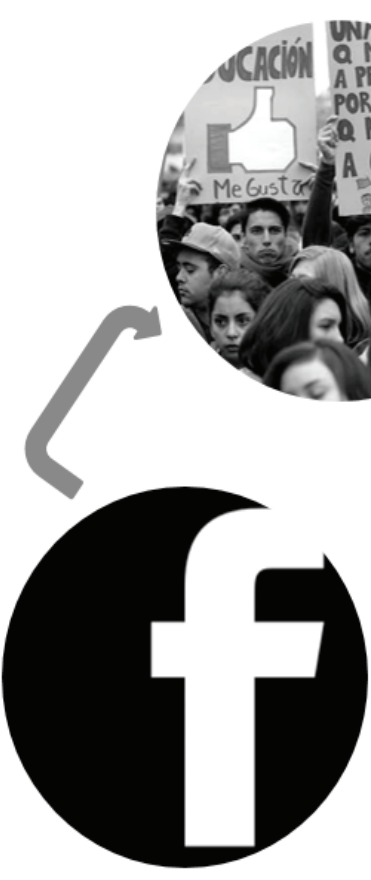

Muestras finales por periodo (2011-2013)

Criterios aplicados a las publicaciones

Tiempo: tres primeros años del último ciclo de movilizaciones (2011-2013).

Cantidad: 15 publicaciones más compartidas en cada trimestre y un máximo de 25 comentarios por entrada.

Temas: entre las 15 publicaciones más compartidas por semestre se eligen aquellas exclusivamente relacionadas con el movimiento estudiantil.

Características lingüísticas/semióticas: se priorizan las publicaciones de carácter multimodal, pues se constata con rapidez que este tipo de discursos alcanza una frecuencia destacada en el total de publicaciones.

\section{Criterios aplicados a las cuentas}

Creadas al inicio de las movilizaciones en 2011 por colectivo estudiantil.

Se proponen informar sobre el conflicto sin adscripción institucional ni partidista.

Orientadas al segmento etario entre 18 y 24 años.

Se mantienen vigentes en la actualidad.

Fuente: elaboración propia.

en adelante), la cual contaba con más de 90 mil seguidores/as al $2013^{3}$.

\subsection{Plan metodológico}

En esta investigación se optó por una metodología mixta. En primer lugar, se emplearon métodos para procesar cuantitativamente el corpus, con el propósito de organizar y cuantificar los datos obtenidos de El y UI entre 2011 y 2013. Éstos se señalan y se describen sucintamente a continuación.

\footnotetext{
Al 2016, El y Ul cuentan con más de 254.000 y 233.000 seguidores/
} as, respectivamente.

\subsubsection{Método para obtener y organizar los datos: Minería de datos}

La minería de datos abarca distintos procedimientos a través de los cuales se recolectan grandes volúmenes de información y se descubren patrones en ellos. Mediante la programación informática de un recuperador (crawler) se ejecutó un proceso de minería de usos web con el cual se recogieron las publicaciones anuales más populares según los metadatos disponibles sobre las interacciones (me gusta, comentarios y compartir) que los/as seguidores/as generaron en El y UI entre 2011 y 2013. Esta extracción fue posible gracias a la Interfaz de Programación de Aplicaciones 
(API en inglés) disponible en la biblioteca de Facebook. Los datos recuperados de El y UI permitieron describir los flujos de uso y circulación de la información, por un lado, así como su progresión y variación a través de los años en estudio, por el otro.

\subsubsection{Método para presentar y cuantificar los datos: Visualización de datos}

Se desarrollaron distintas visualizaciones (gráficos, nubes de palabras e infografías) que ayudaron a representar ciertas relaciones existentes en los volúmenes de información recolectados. Su aplicación para agrupar y cuantificar los datos extraídos de El y UI consistió en mostrar ciertas frecuencias y asociaciones sobresalientes, como por ejemplo los tipos de publicaciones con mayor capacidad de viralización, las interacciones preferidas de los/as seguidores/as, sus evoluciones longitudinales, entre otras.

En segundo lugar, se emplearon métodos para procesar cualitativamente el corpus, con el propósito de elucidar y analizar los discursos producidos y difundidos en El y UI entre 2011 y 2013. Éstos se señalan y se describen sucintamente a continuación.

\subsubsection{Método para elucidar los datos: Enfoque etnográfico en redes sociales}

Dado que la manera de conocer las cuentas consideradas fue dándoles "me gusta" para poder acceder ellas, fue necesario hacerse parte de ambas comunidades online desde el primer momento de la investigación. Con ello fue posible iniciar una observación sistemática de El y UI, y elaborar un archivo donde se relevaron manualmente aquellas publicaciones que resultaron especialmente destacadas y llamativas en virtud de ciertos hitos movilizatorios y comunicacionales que se consignaron en una línea de tiempo. Esto permitió realizar un seguimiento "en tiempo real" de los ciclos de movilización estudiantil, lo que facilitó una mejor contextualización de los datos minados.

\subsubsection{Método para analizar los datos: Estudio crítico del discurso en redes sociales}

Las pautas que se siguieron para elaborar el modelo de análisis se desprenden, fundamentalmente, del estudio piloto que se realizó con diferentes muestras del periodo en estudio (2011-2013). De este modo, los resultados preliminares orientaron la indagación teórica y la selección de las categorías que componen el modelo provisorio. Éste contempla cuatro niveles complementarios de análisis: estructural e interactivo, multimodal, ideológico y epistémico. A su vez, a cada nivel le corresponden tres categorías, doce en total, de las cuales seis son distintivas de cada tipo de práctica identificada, y las otras seis son comunes a ambas. En la sección siguiente se desarrollan estos aspectos con mayor detalle.

\section{Prácticas discursivas estudiantiles en redes sociales}

Como ya se ha adelantado, la identificación de las prácticas discursivas investigadas no procede de una distinción a priori, sino que surge de la retroalimentación entre las teorías exploradas y los resultados arrojados por el estudio piloto. Esto ayuda a relevar ciertas tendencias en la publicación de contenidos en redes sociales, que tienen que ver con el manejo de la información circulante sobre las 
movilizaciones estudiantiles, por un lado, y con el manejo de producciones propias que dan cuenta de asuntos sensibles para el movimiento desde la perspectiva de sus protagonistas, por el otro. De esta forma, se procede a denominar ambas prácticas con el propósito de derivar estos patrones de los usos comunicativos que resultan transversales a las dos comunidades estudiantiles analizadas.

Así, mientras la práctica de gestión contrainformativa abarca ciertas regularidades relacionadas con la reposición y la discusión de noticias sobre el movimiento, la práctica de expansión transmedia engloba ciertas regularidades relacionadas con la elaboración y la difusión de textos de autoría juvenil que dinamizan por la red representaciones relevantes para la identificación del colectivo. La primera práctica es característica de los meses en que irrumpen las movilizaciones, aun cuando acaba asentándose y permanece durante los años posteriores, perdiendo centralidad. La segunda práctica se vuelve notoria una vez que el ciclo movilizatorio ha experimentado cierto grado de maduración, esto es, cuando los/as estudiantes comienzan a avanzar desde acciones de protesta más bien contestatarias a otras más bien creativas.

\subsection{Práctica de gestión contra-informativa}

La representación de los movimientos sociales constituye una práctica mediatizada que es objeto de permanentes disputas en la esfera pública, de allí que la dependencia que se establece entre medios y movimientos sea siempre conflictiva. La cobertura periodística influye en la formación de representaciones sociales que tienden a criminalizar las movilizaciones y sus actores principales (Pérez 2012; Montecino 2011; Aguilera 2008; McLeod
2007; Boyle et al. 2005; Klandermans y Goslinga 1999; Shoemaker 1984; Cohen 1973; Murdock 1973). En este contexto, la utilización de Internet ha transformado los procesos verticales de comunicación (Castells 2014, 2012, 2009), al tiempo que ha supuesto una serie de grietas en el control de la información (Rovira Sancho 2015, 2013a, 2013b). Así, los medios tradicionales que persiguen una hetero-representación negativa de los movimientos, se confrontan en la actualidad a los ciudadanos que usan las tecnologías para promover una auto-representación positiva de su participación política.

Según se ha investigado previamente (Cárdenas 2014b, en prensa), las interacciones comunicativas de los/as jóvenes en redes sociales se configuran en un espacio estructurado por flujos informativos y contrainformativos que pugnan por delimitar el sentido del conflicto educativo. De un lado, se encuentran los discursos periodísticos de la prensa controlada por los grupos políticos y económicos, a cuyo cargo está la mayor parte de los portales de noticias del país; de otro lado, agrupaciones estudiantiles con presencia en redes sociales como Facebook seleccionan, reponen y valoran dichas noticias con el propósito de abrir un espacio de discusión para sus seguidores/as.

En la Figura 3 se ejemplifica cómo se lleva a cabo típicamente la reposición noticiosa en el Facebook de El. La publicación se realiza el 2 de diciembre del 2011 y se compone de: a) una entrada que corresponde a la nota "Estudiantes interrumpen discurso de Piñera y fueron sacados del teatro" tomada del portal online del periódico Publimetro, y; b) una sección de 160 comentarios escritos por sus seguidores/as, de los cuales sólo se muestra una cantidad limitada 
Figura 3. Ejemplo de gestión contra-informativa

Estudiantes Informados compartió un enlace.

2 de diciembre de 2011

Un grupo reducido de personas aprovechó la intervención del Presidente Sebastián Piñera en la Teletón para protestar en el marco del conflicto entre el gobierno y estudiantes.

Según se escuchó en la transmisión en vivo de la televisión, la gente comenzó a gritar consignas en favor del movimiento de universitarios y secundarios, pero fueron rápidamente desalojados del teatro.

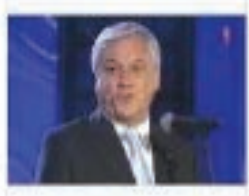

Estudiantes interrumpen discurso de Pin̈era y fueron sacados del teatro - Publimetro

waw. publimetro c

Un crupe reducido de personas aprovectio ta intervencobn del

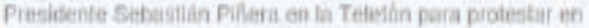

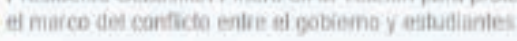

323 me gusta 160 comentarios 40 compartir

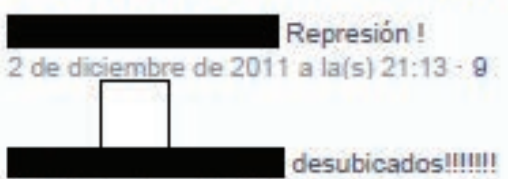

2 de diciembre de 2011 a la(s) 21:13 - 9

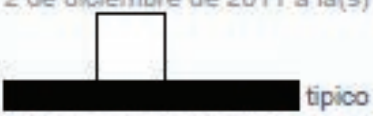

2 de diciembre de 2011 a la(s) 21:13

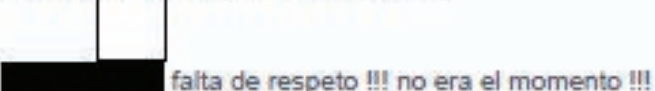

2 de diciembre de 2011 a la(s) 21:13 - 15

Hay momentos y momentos. Y éste no era el momento.

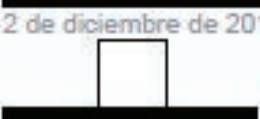

si llamar la atención debes, hacer cualquier cosa es aceptada

2 de diciembre de 2011 a la(s) $21: 14 \cdot 4$

pffff...no faltan.....los sacaron en tipo 1..jajaja

2 de diciembre de 2011 a la(s) 21:14

Grandes.

2 de diciembre de 2011 a la(s) 21:14 - 2

Fuente: Facebook de Estudiantes Informados. 
con fines ilustrativos. A grandes rasgos, la noticia informa sobre la manifestación que realizó un grupo de estudiantes durante el programa televisivo Teletón (instancia que reúne fondos para niños/as discapacitados/as del país), justamente cuando el ex presidente Sebastián Piñera llevaba a cabo el discurso inaugural de la jornada. La publicación fue la más popular del mes, alcanzando 323 me gusta y 40 compartir.

Si bien son diversos los aspectos que pueden resultar de interés para un análisis discursivo con enfoque crítico, el modelo provisorio recoge sólo algunos de los más prominentes, ordenados a distintos niveles de construcción de significados:

a) Análisis estructural e interactivo: a este nivel es posible analizar la composición de las publicaciones más populares y las lógicas de interacción que promueven, examinando (1) cuáles son sus temas recurrentes; (2) cuáles participantes y acciones representan, es decir, cuáles agencias discursivas adquieren preponderancia y cuáles eventos y/o circunstancias se relacionan con ellas, y; (3) qué tipo de interacciones se producen entre los/as comentaristas, esto es, cuáles intervenciones activan el debate y cuáles argumentos y/o evaluaciones suscitan.

b) Análisis ideológico: a este nivel es posible analizar cómo se llevan a cabo modos de hetero y auto-representación competitivos en las publicaciones más populares, indagando (1) cómo se configuran los esquemas ideológicos que sintetizan la oposición nosotros/ellos; (2) cuáles son las opiniones personales y las actitudes grupales que se confrontan más frecuentemente, y; (3) cuáles son las identidades políticas, de clase y/o generacionales mediante las cuales se posicionan habitualmente las actorías juveniles.

c) Análisis epistémico: a este nivel es posible analizar cómo se formulan y negocian ciertos saberes en las publicaciones más populares, examinando (1) cuáles son los modelos situacionales y contextuales subyacentes a ellas; (2) cuáles son los conocimientos de fondo común y las presuposiciones que se implican más a menudo, y; (3) cuáles son las memorias históricas que pueden inferirse de la interacción.

En la Tabla 1 se sintetizan estos aspectos, especificando sus principales referentes teórico-analíticos.

Como se observa, la reposición noticiosa, en cuanto género discursivo, constituye una unidad de análisis compuesta por tres etapas esenciales: un segmento textual (habitualmente la bajada o el primer párrafo de la nota); el hipervínculo de la noticia, a partir del cual se accede a su titular y una imagen referencial, y; la sección de comentarios. Dicha reposición se desarrolla bajo criterios de impacto y economía, dado que se eligen sólo ciertos fragmentos de noticias cuyos contenidos usualmente construyen representaciones polémicas sobre la acción política del movimiento estudiantil, opción que puede responder a la asignación de relevancia que efectúan los/as administradores/ as de El para determinar aquellas publicaciones que pueden generar mayores reacciones (me gusta, comentar, compartir) por parte de sus seguidores/as. Por tanto, la selección de las noticias repuestas afecta simultáneamente la estructura de interacción de los/as comentaristas, cuyas intervenciones, en la mayoría de los casos, aluden directamente al contenido informativo. 
Tabla 1. Propuesta provisoria para el análisis de prácticas de gestión contra-informativa

Niveles de análisis

\begin{tabular}{|c|c|c|c|c|c|}
\hline \multicolumn{2}{|c|}{ Estructural e interactivo } & \multicolumn{2}{|c|}{ Ideológico } & \multicolumn{2}{|c|}{ Epistémico } \\
\hline Categorías & Referentes & Categorías & Referentes & Categorías & Referentes \\
\hline Temas & $\begin{array}{c}\text { Van Dijk (2010a, } \\
2002,1990)\end{array}$ & $\begin{array}{l}\text { Esquema } \\
\text { ideológico }\end{array}$ & $\begin{array}{c}\text { Van Dijk (2009, } \\
\text { 1999) }\end{array}$ & $\begin{array}{c}\text { Modelos } \\
\text { situacionales y } \\
\text { contextuales }\end{array}$ & $\begin{array}{c}\text { Van Dijk } \\
(2012,2011)\end{array}$ \\
\hline $\begin{array}{l}\text { Participantes } \\
\text { y acciones }\end{array}$ & $\begin{array}{c}\text { Van Leeuwen } \\
(2008,1996)\end{array}$ & $\begin{array}{l}\text { Actitudes y } \\
\text { opiniones }\end{array}$ & $\begin{array}{c}\text { Van Dijk (2009, } \\
\text { 1999) }\end{array}$ & $\begin{array}{l}\text { Conocimiento de } \\
\text { fondo común y } \\
\text { presuposiciones }\end{array}$ & $\begin{array}{c}\text { Van Dijk } \\
(2014,2008)\end{array}$ \\
\hline $\begin{array}{l}\text { Interacciones } \\
\text { y tadiscursos }\end{array}$ & $\begin{array}{c}\text { Heisler, Vincent y } \\
\text { Bergeron (2003); } \\
\text { Ilie (2002); } \\
\text { Martínez (2009) }\end{array}$ & Identidades & $\begin{array}{l}\text { Bamberg, De } \\
\text { Fina y Schiffrin } \\
\text { (2011); De Fina } \\
\text { (2011, 2006); } \\
\text { Van Dijk (2010b) }\end{array}$ & $\begin{array}{l}\text { Memorias } \\
\text { históricas }\end{array}$ & $\begin{array}{c}\text { Achugar } \\
(2011,2008)\end{array}$ \\
\hline
\end{tabular}

Fuente: elaboración propia.

Por este motivo, resulta necesario preguntarse por qué se eligen unos contenidos noticiosos en lugar de otros para llamar la atención y así propiciar la discusión de los/as seguidores/ as. En esta línea, resulta interesante que El prácticamente no introduzca textos propios en las reposiciones y, en su lugar, únicamente emplee fragmentos tomados de la redacción de la noticia tal como aparece en el medio de origen. En este sentido, destaca el carácter estratégico -y comúnmente implícito- de la base tanto ideológica como epistémica en virtud de la cual se modelan los procesos de interpretación e interacción juvenil. Los hallazgos preliminares, en esta dirección, señalan la preeminencia de un macropropósito comunicativo constitutivo de esta práctica de gestión contra-informativa: disentir de los significados impuestos por los discursos mediáticos hegemónicos, mediante la insinuación de finalidades veladas y el descubrimiento conjunto de sus sesgos.

En el ejemplo empleado, esto se manifiesta de dos maneras. En primer lugar, mientras la noticia promueve la negativización de la protesta estudiantil (desestabilización del orden, impertinencia, etc.), la reposición noticiosa se lleva a cabo con la finalidad de abrir un debate en torno a la validez de las tácticas de contestación juvenil (sagacidad, repercusión pública, etc.), por un lado, y a los mecanismos de coerción de las autoridades políticas y policiales (abuso de poder, uso desmedido de la fuerza, etc.), por el otro. En segundo lugar, los comentarios revelan que los/as seguidores/ as de El modelan representaciones sociales 
divergentes sobre la acción política del movimiento estudiantil, recogiendo distintos posicionamientos susceptibles de interpelación directa ("desubicados" / "grandes", "no era el momento..." / "represión", etc.). Estas instancias de discusión, a la larga, estimulan y enriquecen las formas de socialización juvenil, haciendo posible redimensionar continuamente los alcances materiales y simbólicos de sus movilizaciones.

En suma, cuando los/as jóvenes seleccionan, reapropian y recontextualizan los contenidos noticiosos que les resulten relevantes, y los hacen circular al interior de comunidades en las que confluyen búsquedas e intereses similares, están constantemente modificando las posiciones de poder en la comunicación online pues, aun cuando los/as estudiantes tienen un lugar subordinado en la prensa tradicional, en el espacio de negociación política conformado por El dichos roles se alteran, equiparando su influencia. Por ello, esta práctica de gestión contra-informativa no sólo tiende, con el paso del tiempo, a legitimar las acciones colectivas juveniles, sino también a denunciar los intentos estereotipadores que los medios efectúan para criminalizar al movimiento estudiantil. Se obtiene así un cuestionamiento sistemático orientado a desmantelar las versiones periodísticas oficiales, pudiendo confrontarlas o rectificarlas continuamente.

Con ello, se activan dinámicas de interacción que, al no depender ni estar supeditadas a las esferas adultas y poderosas, sirven a los objetivos de minimizar los efectos manipuladores derivados de la representación mediática, por un lado, y de profundizar los sentidos de pertenencia de los/as jóvenes movilizados/ as, por el otro. Las redes sociales devienen así en lugares de diálogo y resignificación, delineándose como emplazamientos prevalentes del discurso político juvenil. Su uso conduce, paulatinamente, a la formación de una ciudadanía crítica, en virtud de la cual los/as estudiantes pueden llegar a expresar nuevas relaciones de fuerza que constituyen en estratégico el control informativo.

\subsection{Práctica de expansión transmedia}

Si bien la gestión contra-informativa se presenta como una práctica discursiva habitual en redes sociales, a medida que se extienden las movilizaciones estudiantiles se observa la utilización cada vez más sistemática de discursos de elaboración propia que delinean con mayor amplitud las acciones comunicativas características del reciente ciclo movilizatorio. La particularidad de estos discursos reside en que mantienen interesantes relaciones entre sí, es decir, aluden a temáticas, participantes y/o referentes comunes cuyas representaciones confluyen y se expanden a través de la Web Social. Tales usos permiten postular que los/ as jóvenes construyen determinados nodos de significación que son trabajados a lo largo del tiempo, y que sirven principalmente a los propósitos de enmarcado para la acción y la identificación colectiva.

Los trabajos de Jenkins (2008) acerca de la cultura de la convergencia ayudan a delinear los procesos comunicativos a partir de los cuales coinciden los flujos de contenidos provenientes de múltiples plataformas mediáticas. Este fenómeno es sintetizado por el autor bajo la noción de narración transmedia, que Scolari $(2013,2009)$ relaciona estrechamente con la noción de multimodalidad (Kress 2010; Kress y Van Leeuwen 2006, 2001; Van Leeuwen 2005). 
Por su parte, Lehtonen (2000) retoma este carácter multimodal de la cultura contemporánea para enlazarlo a los procesos de intermedialidad que definen los usos comunicativos online. Estas conexiones entre discursos que pueden ser recuperados transversalmente en la web explican la continuidad entre los textos generados por los/ as jóvenes durante sus movilizaciones, así como sus usos, sentidos e impactos en coyunturas de conflicto y cambio social.

En este contexto, Costanza-Chock (2013, 2012, 2010) propone la noción de movilización transmedia para explicar las prácticas comunicativas, políticas y culturales llevadas a cabo por los manifestantes con el fin de transformar la conciencia ciudadana en escenarios de disputas por el poder. Para este autor, mediante estas estrategias transmediales se articulan múltiples discursos multimodales elaborados colaborativamente, los cuales se despliegan de unos medios a otros, conformando una narrativa coherente que representa el mundo del movimiento social. Esta propuesta puede ser abordada discursivamente gracias a las aportaciones de Lemke (2013, 2009a, 2005), cuya noción de recorridos transmedia se enfoca en el análisis de las redes intertextuales que cruzan las fronteras de los géneros discursivos y las plataformas tecnológicas donde los textos aparecen.

En las cuentas de El y UI analizadas circulan numerosos discursos de autoría juvenil que buscan llamar la atención sobre sus causas, demandas y reivindicaciones grupales, explotando diversas modalidades semióticas en combinación. Sobresalen así publicaciones de afiches, memes, caricaturas, infografías y fotografías de lienzos, carteles, rayados e intervenciones callejeras que destacan por su capacidad de visibilizar los repertorios de acción e interacción estudiantil en espacios simultáneos de apropiación y resistencia. De esta manera, los discursos juveniles circulan de unos lugares a otros - de la calle a las redes sociales-, y de unas prácticas sociales a otras de las convocatorias online a las funas ${ }^{4}$ o tomas ${ }^{5}$ en recintos públicos-, constituyendo no sólo una estrategia comunicativa de alto impacto sino que, además, desvelando los complejos procesos de producción y transposición de significados que aseguran la mayor notoriedad posible de su movilización.

La Figura 4 reúne un conjunto de publicaciones efectuadas entre el 20 de junio de 2011 y el 4 de diciembre de 2013 en el Facebook de UI. Todas ellas tienen como referente principal la serie animada Pokémon, transmitida en Chile entre 1994 y 2004 por el canal de televisión abierta Chilevisión. Como se aprecia de izquierda a derecha, tanto el cómic, como el videojuego y el afiche político recurren a su argumento y a algunos de sus personajes con distintas finalidades de protesta. A grandes rasgos, en el primer caso se critica la mercantilización de derechos sociales básicos, como ocurre con la salud $-\mathrm{y}$, por extensión, en Chile, con la educación-; en el segundo caso, se critica al ex presidente Sebastián Piñera ("wild Piñera appeared"), quien cuenta como el adversario (político) al cual se debe derrotar; en el tercer caso, se convierte a una de sus figuras animadas más emblemáticas en ícono revolucionario (Pikachu como Che Guevara), cuyo ejemplo es presentado a los/as estudiantes como un modelo a seguir.

Protestas que buscan encarar a ciertas figuras políticas cuando éstas desarrollan actividades públicas.

Ocupación física de los espacios públicos. 
Figura 4. Ejemplo de expansión transmedia en redes sociales
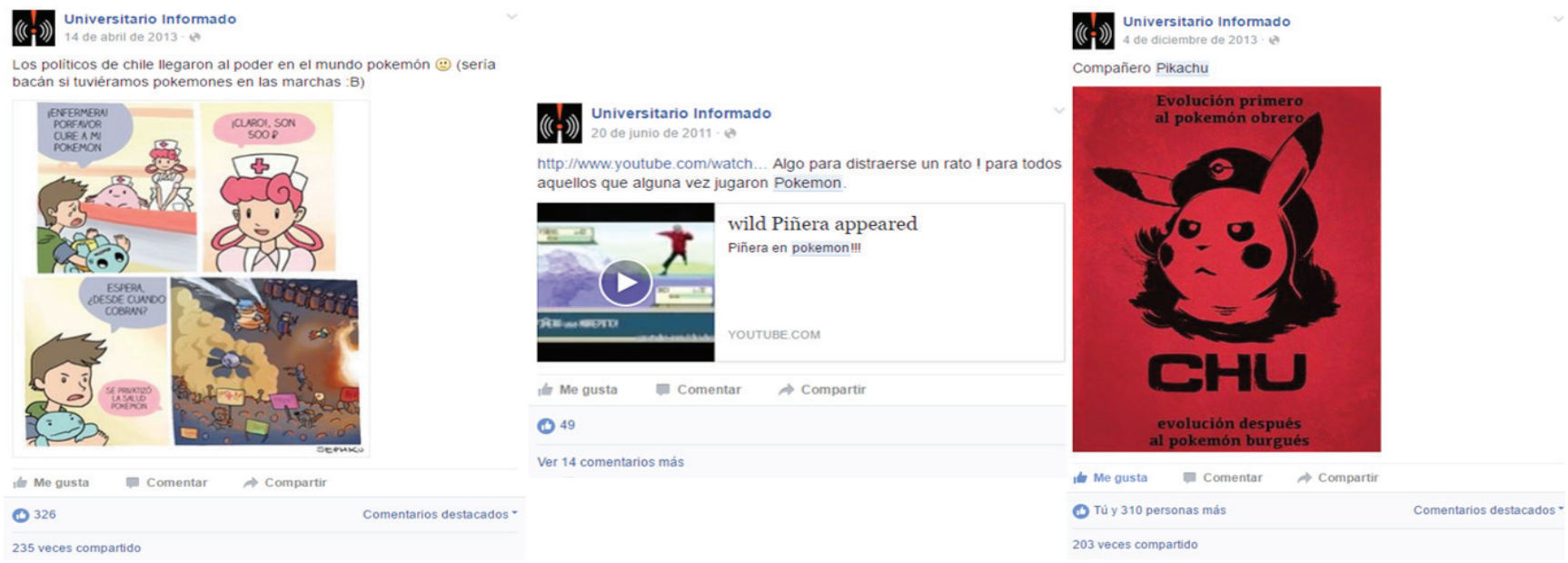

Fuente: Facebook de Universitario Informado.

Si bien son numerosos los aspectos que resultan de interés para un análisis discursivo con enfoque crítico, el modelo provisorio recoge sólo algunos de los más prominentes, ordenados a distintos niveles de construcción de significados:

a) Análisis multimodal: a este nivel es posible analizar (1) cómo se construyen significados que representan, narrativa o conceptualmente, a los actores sociales, los eventos en los que se involucran y sus circunstancias respectivas; cómo se construyen los significados interactivos que dan cuenta de la relación que se establece entre la realidad representada y sus observadores/ as (en este caso, los/as seguidores/as de la red social), y; cómo se construyen los significados composicionales derivados de la organización de los elementos al interior de las imágenes publicadas. Dado que uno de los principales rasgos de varios de estos discursos es su carácter simbólico, también es posible analizar (2) cómo se construyen determinadas metáforas multimodales, es decir, cómo la representación visual y textual de los/as participantes, acciones y/o conceptos involucrados generan efectos retóricos y estéticos particulares. Además, puesto que gran parte de estos discursos recuperan hitos movilizatorios familiares, sensibles o controvertidos, cabe la posibilidad de analizar (3) cómo se construyen ciertas emociones que refuerzan procesos de afiliación, pertenencia y solidaridad grupal por parte de los actores juveniles.

b) Análisis ideológico: estas publicaciones, a su vez, modelan procesos de oposición ideológica (esquema nosotros $\mathrm{v} / \mathrm{s}$ ellos) que son transversales a temas y/o referentes recurrentes, por lo que es posible analizar (1) 
cómo se construyen modos de hetero y autorepresentación competitivos en base a (2) opiniones personales y actitudes grupales en torno a la realidad representada, e (3) identidades políticas, de clase y/o generacionales a las que remiten estos discursos en su conjunto.

c) Análisis epistémico: a este nivel es posible analizar (1) cuáles son los modelos mentales (situacionales y contextuales) que comparten estos discursos y facilitan su asociación, (2) cuáles son los conocimientos de fondo común y las presuposiciones que se desprenden de ellos, y (3) cuáles son las memorias que sitúan la acción juvenil en un horizonte compartido de participación histórica.

En la Tabla 2 se sintetizan estos aspectos, especificando sus principales referentes teóricoanalíticos.

Tal como se observa en el ejemplo, este tipo de expansión no sólo remite a un típico caso de narración transmedia dado por la utilización de

Tabla 2. Propuesta provisoria para el análisis de prácticas de expansión transmedia

\begin{tabular}{|c|c|c|c|c|c|}
\hline \multicolumn{6}{|c|}{ Niveles de análisis } \\
\hline \multicolumn{2}{|c|}{ Multimodal } & \multicolumn{2}{|c|}{ Ideológico } & \multicolumn{2}{|c|}{ Epistémico } \\
\hline Categorías & Referentes & Categorías & Referentes & Categorías & Referentes \\
\hline $\begin{array}{l}\text { Significados } \\
\text { representacionales, } \\
\text { interactivos y } \\
\text { composicionales }\end{array}$ & $\begin{array}{c}\text { Kress y Van Leeuwen } \\
(2006)\end{array}$ & $\begin{array}{l}\text { Esquema } \\
\text { ideológico }\end{array}$ & $\begin{array}{c}\text { Van Dijk (2009, } \\
\text { 1999) }\end{array}$ & $\begin{array}{c}\text { Modelos } \\
\text { situacionales y } \\
\text { contextuales }\end{array}$ & $\begin{array}{c}\text { Van Dijk } \\
(2012,2011)\end{array}$ \\
\hline $\begin{array}{l}\text { Metáfora multimodal } \\
\text { y representación } \\
\text { visual }\end{array}$ & $\begin{array}{c}\text { Feng y Espindola } \\
\text { (2013); Feng y } \\
\text { O'Halloran (2013); } \\
\text { Forceville y Urios- } \\
\text { Aparisi (2009); Pardo } \\
\text { Abril }(2013,2012 b)\end{array}$ & $\begin{array}{l}\text { Actitudes y } \\
\text { opiniones }\end{array}$ & $\begin{array}{c}\text { Van Dijk (2009, } \\
\text { 1999) }\end{array}$ & $\begin{array}{l}\text { Conocimiento de } \\
\text { fondo común y } \\
\text { presuposiciones }\end{array}$ & $\begin{array}{c}\text { Van Dijk } \\
(2014,2008)\end{array}$ \\
\hline $\begin{array}{l}\text { Metáfora multimodal } \\
\text { y prosodia emocional }\end{array}$ & $\begin{array}{l}\text { Kövecses (2000); } \\
\text { Feng y Qi (2014) }\end{array}$ & Identidades & $\begin{array}{c}\text { Bamberg, De } \\
\text { Fina y Schiffrin } \\
\text { (2011); De Fina } \\
\text { (2011), De } \\
\text { Fina, Schiffrin y } \\
\text { Bamberg (2006); } \\
\text { Van Dijk (2010b) }\end{array}$ & $\begin{array}{l}\text { Memorias } \\
\text { históricas }\end{array}$ & $\begin{array}{c}\text { Achugar } \\
(2011,2008)\end{array}$ \\
\hline
\end{tabular}

Fuente: elaboración propia. 
una trama y unos protagonistas reconocibles, sino que, además, recurre a unos productos de la cultura popular que constituyen un claro referente generacional para los actores movilizados ${ }^{6}$. Esta predisposición promueve una atmósfera de complicidad, juego y compañerismo que potencia efectos cognitivos y emocionales indispensables para la alineación ideológica e identitaria. De esta manera, la protesta estudiantil toma forma y se fortalece a partir de una serie de elementos simbólicos que optimizan los procesos de intersubjetividad juvenil.

Uno de los rasgos más sobresalientes de este caso de expansión transmedia dice relación con los usos humorísticos, satíricos e irónicos presentes en las publicaciones, en razón de los cuales se cuestiona el modelo de sociedad neoliberal, la autoridad institucional y la desigualdad de clases, transitando de modalidades de protesta tradicionales (contestación directa) a otras más bien transgresoras (mediadas por la creatividad). Esta dislocación le permite a los/as jóvenes postular modos alternativos de enmarcar la crisis social, subvirtiendo los modos convencionales de ejercer la crítica política.

La práctica de expansión transmedia no sólo garantiza la visibilización de los cuestionamientos estudiantiles, sino que, a su vez, pone de manifiesto que los puntos de vista predominantes pueden ser constantemente tensionados por una oposición dialógica implícita (Lemke 2003), generalmente con una orientación lúdica o jocosa. Esto otorga a los/as jóvenes la posibilidad de romper el monologismo impuesto por las autoridades adultas, "incluyendo múltiples voces sociales, y dando (...) acceso al campo

Un ejemplo similar puede encontrarse en el flashmob "Genkidama por la Educación" basado en la serie animada Dragon Ball Z. Para un análisis de este caso ver García y Aguirre (2016). de la heteroglosia, a la diversidad de discursos y al conflicto" (Lemke 2003: 323). Por tanto, estas construcciones incrementan las perspectivas interpretativas disponibles, lo que "tiende a asegurar que pocas de las naturalizaciones incrustadas por los poderosos permanezcan intactas" (Lemke 2009a: 593).

De ello se desprende que, a partir la correlación de diferentes publicaciones, sea posible seguir o, más concretamente, leer ciertas cadenas semánticas transversales a los discursos multimodales que replican y amplifican sentidos relevantes para la representación de la acción política juvenil. Por ello, estos discursos constituyen medios privilegiados para sintetizar narraciones que fundamentan los procesos de identificación de los/as estudiantes movilizados/as.

Dicho esto, es posible sintetizar que esta práctica se caracteriza por disputar una autorepresentación positiva de los/as actores juveniles, perfilándolos como interlocutores con derecho a crítica y contestación permanente. Según se ha investigado previamente (Cárdenas 2014c), esta práctica traza particulares conexiones entre significados que convergen a través de los textos y las redes donde éstos son apropiados y difundidos, delineando diversas dimensiones representacionales donde los/as jóvenes intervienen como comunicadores políticos, diseñadores de mensajes y mediadores de conocimiento (Cárdenas 2014d). Habitualmente, dichos recorridos se construyen a partir de ciertas coyunturas mediáticas ${ }^{7}$ que, a la larga, tienen el potencial de convertirse en hitos comunicacionales

Otro caso de expansión transmedia estudiado se configura a partir de la denominación "inútiles subversivos" (Cárdenas 2014c), efectuada por el ex senador de derecha Carlos Larraín en las declaraciones que ofrece a la prensa tras la marcha no autorizada del 4 de agosto de 2011. Para un análisis de la cobertura mediática de la jornada ver Pérez (2012). 
de gran alcance (Aguilera 2014), los cuales se incorporan al reservorio de la memoria estudiantil, modelando sus acciones presentes y futuras.

\section{Conclusiones}

Este artículo ha buscado identificar, caracterizar y explicar dos prácticas discursivas online producidas por los/as jóvenes chilenos en el contexto de las recientes movilizaciones estudiantiles (2011-2013). Por un lado, la práctica de gestión contra-informativa se relaciona con los modos mediante los cuales los/ as jóvenes administran los contenidos noticiosos sobre su protesta, especialmente aquellos que propenden a su hetero-representación negativa. Al respecto, se constata cómo los/ as jóvenes resisten continuamente la cobertura de los medios de comunicación tradicionales, disputando la conformación de representaciones sociales críticas acerca de su acción colectiva, específicamente de aquellas que los niegan, marginan y excluyen de los procesos de transformación política. A partir de una reorientación ideológica de las informaciones circulantes en las redes sociales sobre sus manifestaciones, los/as jóvenes modelan una oposición sistemática hacia los grupos dominantes, esto es, confrontan, discuten y negocian los estereotipos que prejuician sus modalidades de figuración y contención pública.
Por otro lado, la práctica de expansión transmedia se constituye en base a los discursos elaborados y difundidos por los/as jóvenes en las redes sociales, los cuales tienen el potencial de gestar, replicar y extender a través del tiempo ciertos nodos de significación a partir de los cuales ellos/as pueden reivindicarse como actores políticos legítimos. Con esto, se estructuran cadenas semánticas articuladas sobre la base de temas, participantes y/o referentes comunes patentes en múltiples diseños multimodales, cuya correlación amplifica efectos socio-cognitivos que estimulan la pertenencia generacional, modelan las creencias grupales y activan emociones relevantes para la identificación colectiva.

De esta manera, ambas prácticas les brindan a los/as jóvenes la oportunidad de posicionar y ampliar sus ejercicios de resignificación, enclaves estratégicos para la consecución de sus causas y sus luchas compartidas. En conjunto, aportan a la reformulación de un paradigma que legitima la educación como derecho universal sustentado en el acceso igualitario y gratuito, adjudican a las nuevas generaciones un rol de cambio protagónico de donde deriva el carácter memorable de su movilización en la historia reciente, y proveen un espacio de visibilización de la acción estudiantil que propone cambios sustantivos en la sociedad chilena de posdictadura. 


\section{Bibliografía}

Achugar, M. 2011. "Aproximaciones discursivas a la transmisión del pasado reciente: Síntesis cualitativa". En (re) construcción: Discurso, identidad y nación en los manuales escolares de historia y de ciencias sociales. Oteíza, T. y Pinto, D. (Eds.). Santiago: Cuarto propio. 43-88.

2008. What we remember. The construction of memory in military discourse. Philadelphia: John Benjamins.

Aguilera, O. 2014. Generaciones: Movimientos juveniles, políticas de la identidad y disputas por la visibilidad en el Chile neoliberal. Buenos Aires: CLACSO.

2012. "Repertorios y ciclos de movilización juvenil en Chile (2000-2012)". Utopía y Praxis Latinoamericana, 17 (57): 101108.

2010a. "Cultura política y política de las culturas juveniles". Utopía y Praxis Latinoamericana, 15 (50): 91-102.

2010b. "Acción colectiva juvenil: De movidas y finalidades de adscripción". Nómadas, 32: 81-98.

2008. "Medios de comunicación en Chile, movimientos juveniles y políticas de la visibilidad". Comunicação, Mídia e Consumo, 5 (14): 55-68.

Bamberg, M., De Fina, A. y Schiffrin, D. 2011. "Discourse and identity construction". Handbook of identity. Theory and research. Schwartz, S. J., Luyckx, K. y Vignoles, V. (Eds.). New York: Springer. 177-199.

Bell, D. 2006. Cybercultures: Cyberculture, cyberpolitics, cybersociety. London-New York: Routledge.

Benford, R. y Snow, D. 2000. "Framing processes and social movements: An overview and assessment". Annual Review of Sociology, 26: 611-639.

Bennett, W. L. y Segerberg, A. 2013. The logic of connective action. Digital media and the personalization of contentious politics. Cambridge: Cambridge University Press.

Boyle, M. et al. 2005. "Newspaper and protest: An examination of protest coverage from 1960-1999". Journalism \& Mass Communication Quarterly, 82: 638-653.

Butler, M. 2011. Clicktivism, slacktivism, or 'real' activism? Cultural codes of American activism in the Internet era. Ann Arbor: Pro Quest.

Cammaerts, B., Mattoni, A. y McCurdy, P. (Eds.). 013. Mediation and protest movements. Bristol-Chicago: Intellect-The University of Chicago Press.

Cárdenas, C. 2014a. "Jóvenes e (in)visibilización histórica: Modos de representación ideológica de la juventud chilena en el pasado reciente (1970-1990)". Signos. Estudios de Lingüística, 47 (85): 217-244.

2014b. "Representación de la acción política de los estudiantes chilenos. Movilización de significados en redes sociales". Última Década, 22 (40): 57-84.

2014c. "Inútiles y subversivos: Representación transmedia de los estudiantes chilenos en redes sociales".
Romanica Olomucensia, 26 (2): 173-190.

2014d. "Representación visual de la movilización estudiantil en Chile: Las fotografías de las marchas como espacios de narración, actuación e identificación política". Onomázein, Número Especial IX ASFAL: 115-137.

2011. "(In)visibilización juvenil: Acerca de las posibilidades de las y los jóvenes en la historia reciente del país". Última Década, 19 (35): 11-31.

En prensa. "Representación online del movimiento estudiantil chileno: Reapropiación noticiosa en Facebook". Estudios Filológicos.

Castells, M. 2014. "El poder de las redes". Vanguardia Dossier, 50: 6-13.

Alianza Editorial.

2009. Comunicación y poder. Madrid: Alianza Editorial. 2007. "Communication, power and counter-power in

he network society". International Journal of Communication, 1 : 238-266.

Cohen, S. 1973. "Mods and rockers: The inventory as manufactured news". The manufacture of news. Deviance social problems and mass media. Cohen, S. y Young, J. (Eds.). London: Constable. 263-279.

Costanza-Chock, S. 2014. Out of the shadows, into the streets! Transmedia organizing and the immigrant rights movement. Cambridge-London: MIT Press.

2013. "Transmedia mobilization in the Popular Association of the Oaxacan Peoples, Los Angeles". Mediation and protest movements. Cammaerts, B., Mattoni, A. y McCurdy, P. (Eds.). Bristol-Chicago: Intellect-The University of Chicago Press. 95-144.

2012. "Mic check! Media cultures and the occupy movement". Social Movement Studies, 11 (3-4): 375-385.

2010. Se ve, se siente: Transmedia mobilization in the Los Angeles immigrant rights movement. Faculty of the USC Graduate School: University of Southern California.

De Fina, A. 2011. "Discourse and identity". Discourse studies: A multidisciplinary introduction ( $2^{\text {nd }} \mathrm{Ed}$.). Van Dijk, T. (Ed.). London: Sage. 263-282.

, Schiffrin, D. y Bamberg, M. 2006. Discourse and identity. Cambridge: Cambridge University Press.

Elleström, L. 2010. "The modalities of media: A model for understanding intermedial relations". Media borders, multimodality and intermediality. Elleström, L. (Ed.). London: Palgrave McMillan. 11-50.

Feixa, C., Fernández-Planells, A. y Figueras-Maz, M. 2016. "Generación Hashtag. Los movimientos juveniles en la era de la web social". Revista Latinoamericana de Ciencias Sociales, Niñez y Juventud, 14 (1): 107-120.

Feixa, C., Pereira, I. y Juris, J. 2009. "Global citizenship and 
the 'new, new' social movements: Iberian connections". Young, 17 (4): 421-442.

Feng, D. y Espindola, E. 2013. "Integrating systemic functional and cognitive approaches to multimodal discourse analysis". I/ha do Desterro, 64: 85-110.

Feng, D. y O'Halloran, K. 2013. "The visual representation of metaphor: A social semiotic approach". Review of Cognitive Linguistics, 11 (2): 320-335.

Feng, D. y Qi, Y. 2014. "Emotion prosody and viewer engagement in film narrative". Narrative Inquiry, 24 (2): 347-367.

Forceville, Ch. y Urios-Aparisi, E. 2009. Multimodal metaphor. Berlin-New York: Mouton de Gruyter.

Gamson, W. y Meyer, D. 1999. "Marcos interpretativos de la oportunidad política". Movimientos sociales: Perspectivas comparadas. McAdam, D., McCarthy, J. y Zald, M. (Eds.). Madrid: Itsmo. 389-412.

Garcés, M. 2012. El despertar de la sociedad. Los movimientos sociales en América Latina y Chile. Santiago: LOM.

García, Ó. y Aguirre, F. 2016. "Spatial practices and narratives: The GenkiDama for Education by Chilean Students". Occupy. The spatial dynamics of discourse in global protest movements. Martín Rojo, L. (Ed.). Amsterdam-Philadelphia: John Benjamins. 157-178.

Heisler, T., Vincent, D. y Bergeron, A. 2003. "Evaluative metadiscursive comments and face-work in conversational discourse". Journal of Pragmatics, 35 (10): 1613-1631.

Herring, S. y Androutsopoulos, J. 2015. "Computer-mediated discourse 2.0". The handbook of discourse analysis (2 $\left.{ }^{\text {nd }} E d.\right)$. Tannen, D., Hamilton, H. E. y Schiffrin, D. (Eds.). Chichester: John Wiley \& Sons. 127-151.

2014. "Research: Computer-mediated communication". En línea, disponible en: http://www.asis.org/Bulletin/Feb-14/ FebMar14_Herring.html (consultado en marzo de 2014). 2013. "Discourse in Web 2.0: Familiar, reconfigured, and emergent". Discourse 2.0: Language and new media. Tannen, D. y Trester, A. M. (Eds.). Washington: Georgetown University Press. 1-26.

2004. "Computer-mediated discourse analysis: An approach to researching online behavior". Designing for virtual communities in the service of learning. Barab, S., Kling, R. y Gray, J. (Eds.). New York: Cambridge University Press. 338-376.

Hill, K. y Hughes, J. 1998. Cyberpolitics: Citizen activism in the age of the Internet. New York: Rowman \& Littlefield.

llie, C. 2002. "Discourse and metadiscourse in parliamentary debates". Journal of Language and Politics, 1 (2): 269-291.

Jenkins, H. 2008. Convergence culture: La cultura de la convergencia de los medios de comunicación. Barcelona: Paidós.

Jordan, T. y Taylor, P. 2004. Hacktivism and cyberwars: Rebels with a cause? London-New York: Routledge.

Klandermans, B. y Goslinga, S. 1999. "Discurso de los medios, publicidad de los movimientos y la creación de marcos para la acción colectiva: Ejercicios teóricos y empíricos sobre la construcción de significados". Movimientos sociales: Perspectivas comparadas. McAdam, D., McCarthy, J. y Zald, M. (Eds.). Madrid:
Itsmo. 442-474.

Kövecses, Z. 2000. Metaphor and emotion. Language, culture, and body in human feeling. Cambridge: Cambridge University Press.

Kress, G. 2010. Multimodality: A social semiotic approach to contemporary communication. London-New York: Routledge. y Van Leeuwen, T. 2006. Reading images. The grammar of visual design. London-New York: Routledge.

2001. Multimodal discourse: The modes and media of contemporary communication. Oxford: Oxford University Press.

ledema, R. 2003. "Multimodality, resemiotization: Extending the analysis of discourse as multi-semiotic practice". Visual Communication, 2 (1): 29-57.

Lehtonen, M. 2000. "On no man's land. Theses on intermediality". Nordicom Information, 3-4: 11-24.

Lemke, J. 2013. "Multimedia and discourse analysis". Routledge handbook of discourse analysis. Gee, J. P. y Handford, M. (Eds.). London-New York: Routledge. 79-89.

2009a. "Transmedia traversals: Marketing meaning and identity". Interdisciplinary perspectives on multimodality: Theory and practice. Baldry, A. y Montagna, E. (Eds.). Campobasso: Palladino. 576-596.

2009b. "Multimodality, identity, and time". Routledge handbook of multimodal analysis. Jewitt, C. (Ed.). London-New York: Routledge. 140-150.

2005. "Critical analysis across media: Games, franchises, and the new cultural order". Approaches to critical discourse analysis. Labarta Postigo, M. (Ed.). Valencia: Universidad de Valencia. 1-22.

2003. "Travels in hypermodality". Visual Communication, 1 (3): $299-325$

2001. "Discursive technologies and the social organization of meaning". Folia Linguistica, 35 (1-2): 79-96.

Machin, D. y Van Leeuwen, T. 2007. Global media discourse: A critical introduction. London-New York: Routledge.

Martín Rojo, L. (Ed.). 2016. Occupy. The spatial dynamics of discourse in global protest movements. Amsterdam-Philadelphia: John Benjamins.

2013. "Paisajes lingüísticos de indignación. Prácticas comunicativas para tomar las plazas". Anuario del conflicto social 2012. Aguilar, S. (Ed.). Barcelona: Observatorio del conflicto social. 275-302.

Martinec, R. y Van Leeuwen, T. 2008. The language of new media design: Theory and practice. London-New York: Routledge.

Martínez, S. 2009. "Argumentation, metadiscourse and social cognition: Organizing knowledge in political communication". Discourse \& Society, 20 (6): 727-746.

McAdam, D., McCarthy, J. y Zald, M. 1999. "Oportunidades, estructuras de movilización y procesos enmarcadores: Hacia una perspectiva sintética y comparada de los movimientos sociales". Movimientos sociales: Perspectivas comparadas. McAdam, D., McCarthy, J. y Zald, M. (Eds.). Madrid: Itsmo. 21-46.

McCaughey, M. y Ayers, M. 2003. Cyberactivism: Online 
activism in theory and practice. London-New York: Routledge.

McLeod, D. 2007. "News coverage and social protest: How the media's protest paradigm exacerbates social conflict". Journal of Dispute Resolution, 143: 185-194.

Montecino, L. 2011. ¿Por qué marchan los estudiantes en Chile?: Discurso electrónico y poder en un ciberperiódico. Cadernos de Linguagem e Sociedade, 12 (2): 179-209.

Muñoz, V. 2011. "Juventud y política en Chile: Hacia un enfoque generacional”. Última Década, 19 (35): 113-141.

Murdock, G. (1973). "Political deviance: The press presentation of a militant mass demonstration". The manufacture of news. Deviance social problems and mass media. Cohen, S. y Young, J. (Eds.). London: Constable. 206-225.

Page, R. et al. 2014. Researching language and social media: A student guide. London-New York: Routledge.

Pardo, N. 2013. "Despojo en la caricatura: Metáfora multimodal". Cadernos de Linguagem e Sociedade, 14: 107-127. 2012a. Discurso en la web. Pobreza en Youtube. Bogotá: Centro Editorial Facultad de Ciencias Humanas, Universidad Nacional de Colombia.

2012b. "Metáfora multimodal: Representación mediática del despojo" Forma y Función, 25 (2): 39-61.

Peña-López, I. 2013. "Casual politics: Del clicktivismo a los movimientos emergentes y el reconocimiento de patrones". Educación Social, 55: 33-51.

Pérez, C. 2012. "The Chilean student movement and the media: A comparative analysis on the linguistic representation of the 04 August, 2011 manifestation in right-wing and left-wing newspapers". Logos. Revista de Lingüística, Filosofía y Literatura, 22 (2): 4-26.

Pickerill, J. 2003. Cyberprotest: Environmental activism online. Manchester: Manchester University Press.

Pino-Ojeda, W. 2014. "Insurgency of discourse and affective intervention: The Chilean students' movement". Argos Aotearoa, 1: 126-135.

Reguillo, R. 2012. Culturas juveniles. Formas políticas del desencanto. Buenos Aires: Siglo XXI.

Rovira Sancho, G. 2013a. "Activismo mediático y criminalización de la protesta: Medios y movimientos sociales en México". Revista Convergencia, 20 (61): 35-60.

2013b. "México, \#YOSOY132: ¡No había nadie haciendo el movimiento más que nosotros!". Anuario del conflicto social 2012. Aguilar, S. (Ed.). Barcelona: Observatorio del conflicto social. 423-448.

Samuel, A. 2004. Hacktivism and the future of political participation. Cambridge: Harvard University.

Scolari, C. 2013. Narrativas transmedia. Cuando todos los medios cuentan. Barcelona: Deusto.

2009. "Transmedia storytelling: Implicit consumers, narrative worlds, and branding in contemporary media production".
International Journal of Communication, 3: 586-606.

Shoemaker, P. 1984. "Media treatment of deviant political groups". Journalism Quarterly, 61 (1): 66-82.

Snow, D. 2004. "Framing processes, ideology, and discursive fields". The Blackwell Companion to social movements. Snow, D., Soule, S. y Kriesi, H. (Eds.). Oxford: Blackwell Publishing. 380412.

et al. 1989. "Frame alignment processes, micromobilization, and movement participation". American Sociological Review, 51 (4): 464-481.

Tarrow, S. 2011. El poder en movimiento. Los movimientos sociales, la acción colectiva y la política. Madrid: Alianza Editorial.

Tascón, M. y Quintana, Y. 2012. Ciberactivismo. Las nuevas revoluciones de las multitudes conectadas. Madrid: Los Libros de la Catarata.

Tilly, Ch. y Wood, L. 2010. Los movimientos sociales, 17682008. Desde sus orígenes a Facebook. Crítica: Barcelona.

Toret, J. (Coord.). 2015. Tecnopolítica y 15M: La potencia de las multitudes conectadas. Un estudio sobre la gestación y explosión del 15M. Barcelona: UOC Ediciones.

. et al. 2013. Tecnopolitica: La potencia de las multitudes conectadas. El sistema red 15M, un nuevo paradigma de la política distribuida. Barcelona: UOC Ediciones.

Van de Donk, W. et al. 2004. Cyberprotest: New media, citizens, and social movements. London-New York: Routledge.

Van Dijk, T. 2014. Discourse and knowledge. Cambridge: Cambridge University Press.

2012. Discurso y contexto. Barcelona: Gedisa.

2011. Sociedad y discurso. Barcelona: Gedisa.

2010a. "Discurso, conocimiento, poder y política.

Hacia un análisis crítico epistémico del discurso". Revista de Investigación Lingüística, 13: 167-215.

2010b. "Political identities in parliamentary debates".

European parliaments under scrutiny. Discourse strategies and interaction practices. Ilie, C. (Ed.). Amsterdam: Benjamins. 29-56. 2009. Discurso y poder. Barcelona: Gedisa.

2008. "Semántica del discurso e ideología". Discurso \& Sociedad, 2 (1): 201-261.

2002. "El conocimiento y las noticias". Quaderns de

Filologia. Estudis de Comunicació, 1: 249-270.

1999. Ideología. Barcelona: Gedisa.

1990. La noticia como discurso. Comprensión, structura y producción de la información. Barcelona: Paidós.

Van Leeuwen, T. 2008. Discourse and practice. New tools for critical discourse analysis. Oxford: Oxford University Press. Routledge. 2005. Introducing social semiotics. London-New York:

1996. "The representation of social actors". Texts and practices. Caldas-Coulthard, C. y Coulthard, M. (Eds.). LondonNew York: Routledge. 32-70. 
$\underline{100}$ 